\title{
Neonatal abstinence syndrome in methadone exposed infants: Role of genetic variability
}

\author{
Andrea L. Fielder \\ Nursing \& Midwifery, Sansom Institute, University of South Australia, Adelaide, SA, Australia \\ J. K. Coller \\ University of Adelaide, Adelaide, SA, Australia
}

M. R. Hutchinson

University of Adelaide, Adelaide, SA, Australia

Ross R. Haslam

Women's \& Children's Hospital, Adelaide, SA, Australia

Nona Lu

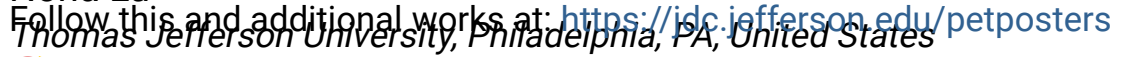

Part of the Pharmacy and Pharmaceutical Sciences Commons beet us knownthow access to this document benefits you

\section{Recommended Citation}

Fielder, Andrea L.; Coller, J. K.; Hutchinson, M. R.; Haslam, Ross R.; Lu, Nona; Adeniyi-Jones, Susan C.; Ehrlich, Michelle; and Kraft, Walter K., "Neonatal abstinence syndrome in methadone exposed infants: Role of genetic variability" (2014). Department of Pharmacology and Experimental Therapeutics Posters. 3. https://jdc.jefferson.edu/petposters/3

This Article is brought to you for free and open access by the Jefferson Digital Commons. The Jefferson Digital Commons is a service of Thomas Jefferson University's Center for Teaching and Learning (CTL). The Commons is a showcase for Jefferson books and journals, peer-reviewed scholarly publications, unique historical collections from the University archives, and teaching tools. The Jefferson Digital Commons allows researchers and interested readers anywhere in the world to learn about and keep up to date with Jefferson scholarship. This article has been accepted for inclusion in Department of Pharmacology and Experimental Therapeutics Posters by an authorized administrator of the Jefferson Digital Commons. For more information, please contact: JeffersonDigitalCommons@jefferson.edu. 
Authors

Andrea L. Fielder, J. K. Coller, M. R. Hutchinson, Ross R. Haslam, Nona Lu, Susan C. Adeniyi-Jones, Michelle Ehrlich, and Walter K. Kraft 


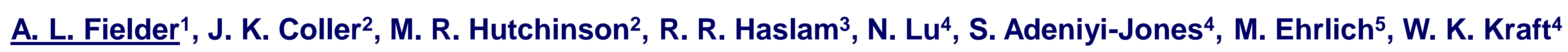

${ }^{1}$ Nursing \& Midwifery, Sansom Institute, University of South Australia, Adelaide, Australia.

${ }^{2}$ School of Medical Sciences, University of Adelaide, Adelaide, Australia. ${ }^{3}$ Women's \& Children's Hospital, Adelaide, Australia. ${ }^{4}$ Thomas Jefferson University, Philadelphia, United States ${ }^{5}$ Mt Sinai School of Medicine, New York, United States.

\section{Opioid dependence in pregnancy}

\section{- Methadone (MD)}

Currently the only FDA/TGA "approved" opioid substitution therapy during pregnancy.

Advantages: $\downarrow$ obstetric complications, $\uparrow$ prenatal care, $\uparrow$ maternal nutrition, $\downarrow$ drug seeking environment.

Disadvantages: Neonatal Abstinence Syndrome (NAS)

\section{Nature vs Nurture}

Dependence $=$ genetics + environment - Infant buccal swab: DNA isolated from !!!!! Uniqueness of current study
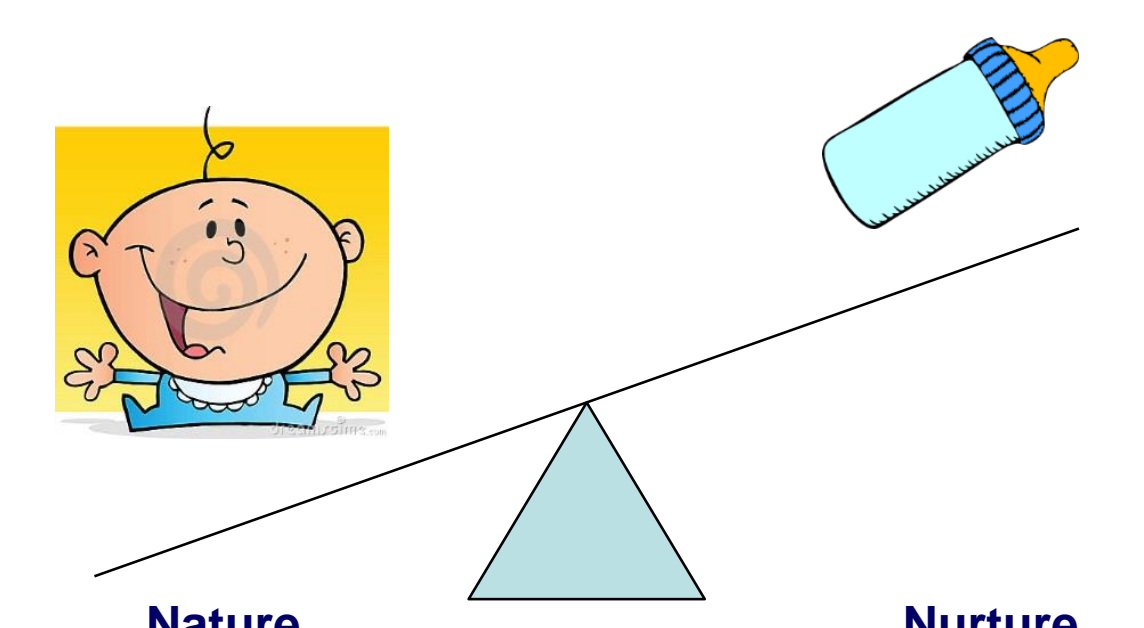

Nature

Nurture

\section{Understanding opioid dependence}

- $\downarrow$ understanding of opioid dependence in adults.

- $\uparrow$ inter-individual variability in response to MD:

- Impact of genetic variability.

- Drug targets/receptors (OPRM1)?

- Drug transporters (ABCB1)

- Metabolising enzymes (CYP2D6) ?

- Immune response (IL-1B)

Glial activation $\rightarrow$ release of immune mediators incl. proinflammatory cytokines interleukin-1 beta (IL-1 $\beta$ ), creates proinflammatory environment $\rightarrow$ neuronal excitability to $\uparrow$ opioid reward and dependence.

\section{Genotyping for OPRM1 and IL-1B}

cheek cells, genotyped

- Genetic markers:

OPRM1 A118G: Wild-type (WT) associated with $\uparrow$ response to opioids

Immune response, IL-1B -31 promoter mutation: Wild-type (WT) associated with $\uparrow$ IL-1 $\beta$ expression

\section{Genetic variability and NAS}

- Despite $\uparrow$ knowledge on the impact of genetic variability on MD response, the underlying mechanisms explaining NAS largely undefined: ? genetic variability.

By assessing genetic variability in mothers and infants, the association between genetic variability and NAS could be used as a predictive tool: $=\uparrow$ management of the infant by potentially $\downarrow$ morphine administered to control NAS.

\section{Aim}

Investigate the impact of IL-1B -31 and OPRM1 A118G genetic variability on NAS incidence (treatment required) \& severity (dose of morphine).

$$
\text { (1) }
$$

Morphine Treatment

Maternal methadone dose at delivery: $\mathrm{mg}$ (median (range))

Gestational age: weeks (median (range))

- Premature (n (\%))

Gender male (n (\%))

Birth weight: $g$ (median (range))

- Low birth weight (n (\%))

Exposure to $(\mathrm{n}(\%))$ :

- Benzodiazepines

- Nicotine

Adjunctive phenobarb (n (\%))
$120(20-220)$

$39(35-41)$

2 (8)

$14(54)$

$2963(2157-3883)$

$5(19)$

$11(42)$

$15(83)$

$6(23)$
- 71 methadone exposed infants; 46 with NAS; 26 with genetic \& morphine treatment data.

No difference in IL-1B or OPRM1 genotypes between infants with \& without NAS, OR (p):

IL-1B: 1.9 (0.21)

OPRM1:0.23 (0.24)

\section{IL-1B -31 Infant genotype (morphine ( $\mathrm{mg})$, median (range))}

\begin{tabular}{|c|c|c|c|c|c|}
\hline WT $(n=21)$ & $>1 \operatorname{Var}(n=5)$ & WT $(n=21)$ & $>1 \operatorname{Var}(n=5)$ & WT $(n=20)$ & $>1 \operatorname{Var}(n=5)$ \\
\hline $0.15(0.1-0.4)$ & $0.2(0.14-0.3)$ & $0.3(0.17-0.66)$ & $0.28(0.23-0.68)$ & $33.8(14.3-79.3)$ & $34.8(26.7-91.8)$ \\
\hline \multicolumn{2}{|c|}{ Initial morphine $(p=0.06)$} & \multicolumn{2}{|c|}{ Max morphine $(p=0.94)$} & \multicolumn{2}{|c|}{ Total morphine $(p=0.67)$} \\
\hline
\end{tabular}

OPRM1 A118G Infant genotype (morphine (mg), median (range))
WT $(\mathrm{n}=24)$
$>1 \operatorname{Var}(\mathrm{n}=2)$
WT $(n=24)$
$>1 \operatorname{Var}(\mathrm{n}=2)$
WT $(\mathrm{n}=23)$
$>1 \operatorname{Var}(\mathrm{n}=2)$
$0.17(0.1-0.4) \quad 0.22(0.13-0.3)$
$0.29(0.17-0.68) \quad 0.24(0.18-0.3)$
Initial morphine $(p=0.73)$
Max morphine $(p=0.39)$

WT $(n=23) \quad>1 \operatorname{Var}(n=2)$
$\begin{aligned} & 34.8(14.3-91.8) \\ & \text { Total morphine }(p=0.38)\end{aligned}$

\section{Conclusion}

- Despite impact of IL-1B and OPRM1 genetic variability on opioid response in adults, this was not observed in infants.

Limitation: small sample size to date.

- This study of possible impact of IL-1B and OPRM1 genetic variability on NAS is ongoing, and could lead to tools to predict NAS incidence \& severity.

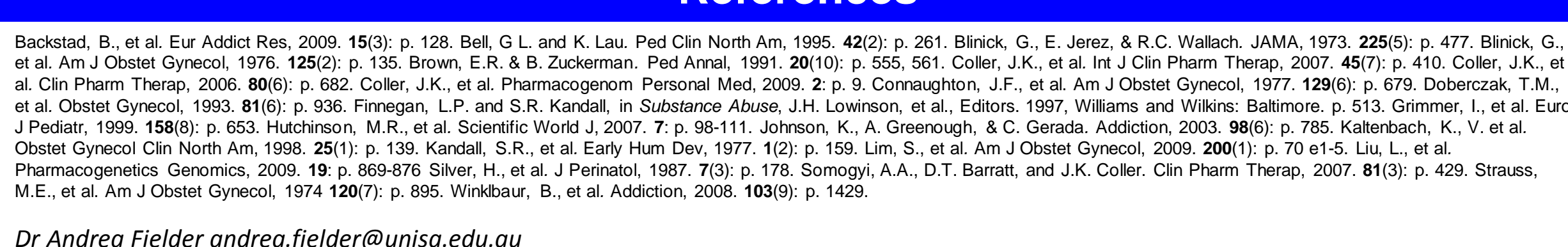

